\title{
CUIDADO DE SI: UN ACTO DE REFLEXIÓN EN EL DESARROLLO PROFESIONAL DEL DOCENTE DE MATEMÁTICA
}

\author{
Ana Duarte Castillo ${ }^{1}$ \\ Elizabeth Gomez Souza ${ }^{2}$
}

\section{Introducción}

Como la mayoría de las matemáticas "dominantes" en nuestro mundo está oculta, los estudiantes necesitan desconstruir críticamente la manera en que esa matemática configura la realidad, para que puedan participar socialmente como ciudadanos informados y críticos en la construcción de una sociedad democrática y socialmente justa para La mayoría de la gente. Un ejemplo de cómo la matemática configura la realidad es:

¿Por qué tiene países que, a pesar de tener reservas petroleras, la energía es muy costosa para la mayoría de las personas?

¿Cómo es el funcionamiento de la distribución de alimentos en la región donde vivimos?

Para poder desconstruir la configuración antes mencionada, es necesario un tipo de profesor, en este caso un tipo de profesor de matemáticas. Es necesario un profesor reflexivo, capaz de examinar y reexaminar, regular y modificar constantemente tanto su propia actividad pedagógica como su práctica y, sobre todo, a sí mismo, en el contexto de esa práctica profesional. Las palabras clave de estos enfoques sobre la formación del profesorado son reflexión, autorregulación, autoanálisis, autocrítica, toma de conciencia, autoformación, autonomía.

Teniendo que para Michel Foucault, ser crítico es una manera de indicar en qué tipo de asunciones, qué tipo de familiar, no desafiado, modos de pensamiento no considerados. Mostrar que las cosas no son tan evidentes como parecen, percibir que lo que hoy es aceptado como evidente no lo será en el futuro (FOUCAULT, 2005). Entre ellos el cuidado de sí, que fue un concepto muy importante en la antigüedad.

La expresión "cuidado de sí mismo" es usada por Foucault para referenciar y traducir una noción compleja y rica que los griegos utilizaban para designar una serie de actitudes ligadas al cuidado de sí mismo, al hecho de ocuparse y de preocuparse consigo que es la de epiméleiaheautoû. Una de las primeras tareas que él plantea es la de separar esta la noción de epiméleaheautoû, de otra noción característica al pensamiento occidental que es la de gnôthi seautón - conocete a ti mismo. La epiméleiaheautoû es, ante todo, una actitud ligada al ejercicio de la política. Es decir, un cierto modo de encarar las cosas, de estar en el mundo, de practicar acciones, de tener relaciones con el otro; una cierta forma de mirar a sí mismo; de acciones que se ejercen de sí, para consigo mismo, que hemos asumido, para modificamos, purificamos, transformamos y transfiguramos.

Un ejemplo de la epiméleiaheautoû, son las cartas de Marco Aurelio (FOUCAULT, 2005) y cómo éste hace su examen matinal de conciencia. Teniendo que el examen de conciencia en la práctica estoica, como también en la práctica pitagórica, tenía dos formas y dos momentos, el examen de la noche, cuando enumeramos los hechos del día para hacer la medición de lo que deberíamos haber hecho; y el examen de la mañana, en que, por el contrario, nos preparamos para las tareas que deben hacer.

\footnotetext{
${ }^{1}$ Doutoranda em Educação em Ciências Matemáticas (UFPA), recebida em Convênio pela FE-Unicamp. Maestria en Educación, Enseñanza de la Matemática (UPEL, Venezuela, 2013). Professora da Universidad Nacional Aberta de Caracas-Venezuela (UNA). E-mail: duarteann@gmail.com.

${ }^{2}$ Pós-Doutoranda em Educação (FE-Unicamp). Doutora em Ensino, Filosofia e História das Ciências (UEFS, 2012). Professora do IEMC-UFPA. E-mail: elizabethmathematics@gmail.com.
} 
Por ello, el objetivo de este escrito académico es analizar el concepto de cuidado de sí, descrito por Foucault como un concepto que debe estar presente en el desarrollo profesional del profesor de matemáticas.

Ahora bien, entre las problemáticas es que durante la vida, y particularmente durante la vida profesional, en este caso la vida profesional de los profesores de matemáticas, independientemente del nivel de la escolaridad en que trabajan, enfrentamos muchos desafíos que generan en nosotros miedos, dudas, delirios, como Eduardo Galeano hace se refiere en el libro Las venas abiertas de América Latina. Muchas veces no estamos seguros de cómo lidiar con los sentimientos que nos invaden, de cómo controlar las pasiones, porque no hay espacios en este caso en las escuelas, ni tiempos para hablar, reflexionar y analizar esos sentimientos como un todo. Y mucho menos analizar y reflejar la práctica educativa y la tarea de enseñanza como un todo. Pero tiene experiencias documentadas como el Grupo de Sábado (GdS) en el contexto de Brasil, es espacios de discusión, donde no sólo están profesores de enseñanza Fundamental, campana investigadores de la universidad. El GdS es una comunidad de aprendizaje docente que fue fundada en 1999, de la que se profundizó más adelante.

Pero, esto espacios no se logran de la noche en la mañana, es necesario que los profesores tengan conciencia de su importancia para mejorar la práctica pedagógica; para lo cual Freire (1996) hace referencia a los siguientes:

la lucha de los profesores en defensa de sus derechos y su dignidad debe ser entendida como un momento importante de su práctica docente, como práctica ética. No es algo externo a la actividad de enseñanza, sino algo intrínseco a ella. La lucha en favor de la dignidad de la práctica docente es tan parte de ella como el respeto que el profesor debe tener a la identidad del alumno, a su persona, a su derecho a ser... (FREIRE, 1996, p. 64)

Por lo tanto, una de las muchas luchas es conseguir espacios de reflexión, formación, análisis, síntesis de la práctica docente. Pensar en la tesis que estoy desarrollando es, inevitablemente, recordar episodios de mi carrera académica que marcaron mi práctica como profesora y mi trabajo en la formación de profesores de matemáticas, donde cada uno de estos episodios ha sido repleto de obstáculos, miedos, inseguridades, además de muchos cuestionamientos filosóficos, políticos; pero al mismo tiempo un gusto de enseñar y una conciencia y dignidad de mi tarea educativa. Como escribe Freire:

la práctica educativa es algo muy serio. Tratamos con personas, con niños, adolescentes o adultos. Participamos de su formación... Estamos intrínsecamente conectados con ellos en su proceso de conocimiento... Podemos contribuir con nuestra responsabilidad, preparación científica y gusto por la enseñanza, con nuestra seriedad y nuestro testimonio de lucha contra la injusticia, para que los aprendices se sienten que se convierten en presencias notables en el mundo. (FREIRE, 1994, p. 52-53)

Es por lo expuesto hasta ahora que ser profesor, pero un profesor progresista, en este caso profesor de matemáticas, no es un trabajo, es un estilo de vida, porque entre las muchas cualidades que debe tener, es la amorosidad. Si no tiene amorosa con sus estudiantes y con el proceso de enseñar, el trabajo no tiene significado. El profesor progresista debe tener un 'amor armado', para poder sobrevivir las prácticas de su quehacer. Como son las injusticias, la indiferencia del poder público, expresada en la desverguenza de los salarios, entre otros (FREIRE, 1994). 


\section{Desarrollo}

Las cualidades deben ser nutridas todos los días. Ser alimentado con humildad, con amor, con valentía, con tolerancia, con determinación, con seguridad, con alegría de vivir, como Freire (1994) refiere en su libro Cartas a quien pretende enseñar y como Foucault (2005) en su libro Hermenéutica del sujeto describe un concepto importante en la antiguiedad, como es Cuidado de sí, haremos referencia específica a la clase 3 de febrero de 1982.

Sin recetas o guiones preestablecidos, como decía el poeta Antonio Machado en su famoso poema Caminante en el hay Camino: Caminante, son tus huellas / el camino y nada más... (MACHADO, s-f)

\section{Cuidado de sí}

En la clase 3 de febrero de 1982, Foucault (2005) hace referencia a diversas cuestiones que tienen como punto de encuentro, el cuidado de sí. Estas cuestiones se refieren a: subjetividad y verdad, cuidado de sí y cuidado de los demás: una inversión de relaciones, la concepción epicurista de la amistad, la concepción estoica del hombre como ser comunitario y la falsa excepción del príncipe.

Ahora bien, al principio de la clase Foucault hace referencia fundamentalmente a Alcibíades, y como Sócrates le habla que debía ocuparse si consigo mismo, para poder gobernar la ciudad, para poder ocuparse con sus conciudadanos, En este apartado, el autor muestra la estrella relación entre el cuidado de sí y la política.

Otro elemento de esta clase es el concepto de cultura que Foucault nos presenta, en un primer momento diversas condiciones que están incluidas en este concepto. Para posteriormente presentar que la cultura es una organización jerárquica de valores, accesible a todos, pero también ocasión de un mecanismo de selección y de exclusión; si llamamos cultura al hecho de que esta organización jerárquica de valores solicita del individuo conductas regadas, costosas, sacrificas, que polarizan toda la vida (FOUCAULT, 2005, p. 21).

En cuanto, las expresiones "prácticas de sí" y "cuidado de sí", están lejos de circunstanciar una especie de apaciguamiento del "sujeto -substancia", de la "perspectiva individual", y nos lanza de forma intensa y reiterada en el campo de las " las relaciones de saber -poder, en las que estamos sin duda involucrados. Para tanto, lejos de sugerir que el sujeto contemporáneo deba volver a sí mismo, a fin de descifrar sus estados de conciencia, características individuales, o incluso, rasgos de deseo, Foucault opone a tal procedimiento otras técnicas de sí -bastante distintas de las prácticas de confesión cristiana y del examen psicológico (o de tipo "psicologizante". Tal es el examen matinal de conciencia que hacía a Marco Aurelio como un cuidado de sí.

Ahora bien, la meditación hecha a Marco Aurelio ¿puede ayudar a partir de narrativas, en la reflexión que el profesor de matemáticas debe construir sobre su práctica? ¿Cómo un examen de conciencia contribuye en el desarrollo profesional del docente de matemáticas, para enriquecer su práctica a partir de reflexiones de su actuación en el proceso pedagógico?

Son interesantes estas interrogantes, porque ponen en evidencia la necesidad de que el profesor de matemática reflexione sobre su quehacer diario.

Estas preguntas son como habla Foucault, momentos en la vida donde la cuestión de saber que se puede pensar diferentemente de lo que se piensa, y percibir diferentemente de lo que se ve, es indispensable para seguir mirando o reflexionando (FOUCAULT, 2005).

Teniendo que las prácticas del cuidado de sí, tienen como objetivo común el de la conversión a sí mismo a partir del principio del bien, que debe ser buscado en el propio sujeto. 
Podríamos resumir este punto en la fórmula de poder, en este caso las relaciones de poder que ejerce el profesor de matemática.

\section{Desarrollo profesional docente}

En la mayoría de los artículos se habla de formación de profesores de matemáticas (FIORENTINI et al, 2002;FIORENTINI 2008, 2012; PONTE, 1992; D`AMBROSIO, 1993). En un trabajo reciente realizado por Fiorentini, Passos y de Lima, 2018, donde presenta los primeros resultados relativos a la primera fase del proyecto de investigación de ámbito nacional titulado Mapeo y estado del arte de la investigación brasileña sobre el profesor que enseña Matemáticas teniendo por objetivo (en el caso de las mujeres, en el caso de las mujeres, en el caso de las mujeres). A partir de los mapeamientos realizados en las 7 regiones, de los 858 trabajos que atendieron a las especificaciones de nuestro corpus, 30385 (35\%) tuvieron como foco sólo la formación inicial de los profesores que enseñan Matemáticas. Pero en muchos, se habla bajo el desarrollo profesional del profesor de matemáticas, entonces cuál es la diferencia entre formación del profesor y desarrollo profesional de profesor de matemáticas? Para dar respuesta a esta pregunta revisamos un artículo que tiene veinte años donde Ponte (1998) hace referencia las diferencias entre formación y desarrollo profesional. En primer lugar, la formación está muy asociada a la idea de "frecuentar" cursos, mientras que el desarrollo profesional ocurre a través de múltiples formas, que incluyen cursos, pero también actividades como proyectos, intercambios de experiencias, lecturas, reflexiones etc.

En segundo lugar, en la formación el movimiento es esencialmente de fuera para dentro, cabiendo al profesor asimilar los conocimientos y la información que le son transmitidos, mientras que en el desarrollo profesional tenemos un movimiento de dentro hacia fuera, correspondiendo al profesor las decisiones fundamentales con respecto a las cuestiones que quiere considerar, a los proyectos que quiere emprender y al modo en que los quiere ejecutar. En tercer lugar, en la formación se atiende principalmente a aquello en que el profesor es carente y en el desarrollo profesional se presta especial atención a sus potencialidades. En cuarto lugar, la formación tiende a ser vista de modo compartimentado, por asuntos o por disciplinas mientras que el desarrollo profesional implica al profesor como un todo en sus aspectos cognitivos, afectivos y relacionales. Finalmente, la formación parte invariablemente de la teoría ya menudo no llega a salir de la teoría, mientras que el desarrollo profesional tiende a considerar la teoría y la práctica de una forma interconectada.

La finalidad del desarrollo profesional es hacer a los profesores más aptos para conducir una enseñanza de la Matemática adaptada a las necesidades e intereses de cada alumno ya contribuir a la mejora de las instituciones educativas, realizándose personal y profesionalmente.

Entonces, cuando hablamos de desarrollo profesional del profesor de matemáticas estamos incluyendo un proceso largo a lo largo de toda la carrera. Además, en el desarrollo profesional hay un importante elemento colectivo y un no menos importante elemento individual. Por un lado, el desarrollo profesional es favorecido por contextos colaborativos (institucionales, asociativos, formales o informales) donde el profesor tiene la oportunidad de interactuar con otros y sentirse apoyado, donde puede conferir sus experiencias y recoger informaciones importantes. No es casual que la realización de un proyecto es, normalmente, una actividad que involucra a todo un grupo de profesores. Uno de los contextos colaborativos más fuerte en Brasil referente a la Formación de profesores es el Grupo de los Sábados, organizado en la UNICAMP por el profesor Dario Fiorentini. 
Fiorentini (2004), al discutir sobre el sentido y modalidades de trabajos colaborativos, cita algunos aspectos presentes en estudios y experiencias con grupos colaborativos: voluntariedad, identidad y espontaneidad; liderazgo compartido o corresponsabilidad de apoyo y respeto mutuo.

En esos contextos colaborativos va a aparecer las narrativas, debido a que son uno de los modos de ordenar las experiencias. Según McEwan y Egan (1998), "la narración es una capacidad humana fundamental, y es por eso que el papel que desempeña en la educación merece más atención" (p. 9). Una narrativa tiene sus raíces en la propia vida de las personas y, por esa razón, es una herramienta que les permite conocerlas y dar sentido a su propia experiencia. Según Chapman (2008), "dado que las historias que contamos reflejan quiénes somos y en lo que podemos transformarnos, ellas pueden proporcionar una base para recuperar, construir o reconstruir los significados de nuestras experiencias" (p. 17).

Este construir y reconstruir es lo que hacía Marco Aurelio en el libro Meditaciones, el fragmento del libro I, es el siguiente.

1. Aprendi com meu avô Verus: o bom caráter e a serenidade.

2. Da reputação e memória legadas por meu pai: o caráter discreto e viril.

3. De minha mãe: o respeito aos deuses, a generosidade e a abstenção não somente do agir mal, como também de incorrer em semelhante pensamento; mais ainda, a frugalidade no regime de vida e o distanciamento do modo de viver próprio dos ricos.

4. Do meu bisavô: o não haver frequentado as escolas públicas e ter desfrutado de bons mestres em casa, e ter compreendido que, para tais fins, é preciso gastar com generosidade.

5. Do meu preceptor: o não ter pertencido à facção nem dos Verdes, nem dos Azuis, nem partidário dos Grandes-Escudos, nem dos Pequenos-Escudos; o suportar as fatigas e ter poucas necessidades; o trabalho com esforço pessoal e a abstenção de excessivas tarefas, e a desfavorável acolhida à calúnia. (MARCO AURELIO, 2011, p. 6).

En la cita anterior podemos mirar cómo Marco Aurelio hacía una reflexión bajo las virtudes de cada una de las personas que estaban en su entorno. Y cada una de las virtudes las incorporaba a su práctica de emperador.

Ahora bien, el uso de narrativas en el proceso de formación de profesores es una tendencia que se apoya principalmente en los trabajos de Clandinin y Connelly $(1995,2000)$. En ese caso, son narraciones producidas por los involucrados en el proceso educativo, sean ellos estudiantes de profesores o profesores en servicio. En este contexto, las narrativas pueden ser consideradas como un medio de socializar las experiencias vividas en las diferentes áreas de formación. El texto escrito abre una instancia de comunicación que posibilita colocar conocimientos, experiencias, tiempos, espacios y sujetos en primer plano con los cuales y en que se constituyó el conocimiento cargado de significados. Estos aspectos del texto narrativo hacen posible entender las experiencias, la situación de la experiencia vivida, las continuidades y las interacciones que ocurren (CLANDININ Y CONNELY, 2000).

\section{Reflexión final}

A partir de este análisis hecho del concepto de sí, particularmente el examen de conciencia que hacía Marco Aurelio, se puede justificar filosóficamente los procesos de reflexión, especialmente en los profesores de matemáticas a partir de narrativas. En el caso de los profesores de matemáticas a partir de las narrativas (CHAPMAN, 2008, CLANDININ Y 
CONNELLY, 2000, RINCÓN Y FIORENTINI, 2008). Y que ellos cambian su práctica pedagógica. Debido a que, el dominio de unas competencias reflexivas para la enseñanza relacionadas con destrezas de reflexión, indagación, autoevaluación, observación sistemática, simulación y colaboración para la mejora de la práctica, frente a otra visión técnica, dominante, y ciertamente más limitada, centrada de manera exclusiva en el dominio de competencias para la intervención

\section{Referencias}

CHAPMAN, O. Narratives in mathematics teach er education. In: TIROSH, D.; WOOD, T. (Ed.), The handbook of mathematics teacher educaction $v .2$ : tools and processes in mathematics teacher education. 2008, p. 15-38.

CLANDININ, D. J.; CONNELLY, F. M. Relatos de experiencia e investigación narrativa. In: LARROSA, J. (Org.). Déjame que te cuente: ensayos sobre narrativa y educación. 1. ed. Barcelona: Laertes, S. A. de Editions

CLANDININ, D. J.; CONNELLY, F. M. Narrative inquiry: experience and story in qualitative research. 2000.

D’AMBROSIO, B. S. Formação de professores de matemática para o século XXI: o grande desafio. Pro-Posições, v. 4, n. 1, p. 10, 1993.

FIORENTINI, D. et al. Formação de professores que ensinam matemática: um balanço de 25 anos de pesquisa brasileira. Educação em Revista. n. 36, p. 137-159, 2002.

FIORENTINI, D. Pesquisar práticas colaborativas ou pesquisar colaborativamente. Pesquisa qualitativa em educação matemática. Belo Horizonte: Autêntica, 2004. p. 47-76.

FIORENTINI, D. A pesquisa e as práticas de formação de professores de matemática em face das políticas públicas no Brasil. Boletim de Educação Matemática, v. 21, n. 29, 2008.

FIORENTINI, D. A formação matemática e didático-pedagógica nas disciplinas da licenciatura em matemática. Revista de Educação PUC-Campinas, n. 18, 2012.

FIORENTINI, D.; PASSOS, C. L. B.; DE LIMA, R. C. R. Mapeamento da pesquisa acadêmica brasileira sobre o professor que ensina matemática. 2018.

FOUCAULT, Michel. La hermeneutica del sujeto: cursos del college de france, 19811982/lectures at the college de france, 1981-1982. Ediciones Akal, 2005.

FREIRE, P. Cartas a quien pretende enseñar. Siglo XXI, 1994.

FREIRE, P. Pedagogia da autonomia: saberes necessários à prática docente. São Paulo: Paz e Terra, 1996.

GAlEANO, E. H. Las venas abiertas de América Latina. Departamento de Publicaciones, Universidad de la República, 1972. 
MARCO AURELIO. Meditaciones. Trad. R. B. Pellicer. Madrid: Gredos, 1990.

MCEWAN, H. Las narrativas en el estudio de la docencia. In: MCEWAN, H.; EGAN, K (Comps.). La narrativa en la enseñanza, el aprendizaje y la investigación. Paraguay: Amorrortu, 1998. p. 236-259.

PONTE, J. P. Concepções dos professores de matemática e processos de formação. 1992.

PONTE, J. P. Da formação ao desenvolvimento profissional. Disponível a, v. 20, 1998.

RINCÓN, J. P. A.; FIORENTINI, D. Aprendizagem docente no estágio transdisciplinar: o caso da Faculdade de Educação da Unicamp. Coleção do V Seminário Nacional de Histórias e Investigações de/em Aulas de Matemática. 2015. 